\title{
Within You / Without You: Biotechnology, Ontology, and Ethics
}

\author{
Daniel P. Sulmasy, OFM, MD, PhD ${ }^{1,2}$ \\ 'John J. Conley Department of Ethics, St. Vincent's Hospital-Manhattan, New York, NY, USA; ${ }^{2}$ New York Medical College, Valhalla, NY, USA.
}

As Implantable Cardioverter Defibrillators (ICDs) have become more common, ethical issues have arisen regarding the deactivation of these devices. Goldstein et al., have shown that both patients and cardiologists consider ICD deactivation to be different from the discontinuation of other life-sustaining treatments. It cannot be argued ethically that ICDs raise new questions about the distinction between withholding and withdrawing treatment, and neither the fact that they are used intermittently, nor the duration of therapy, nor the mere fact that they are located inside the body can be considered unique to these devices and morally decisive. However, frequent allusions to the fact that they are located inside the body might provide a clue about what bothers patients and physicians. As technology progresses, some interventions seem to become a part of the patient as a unified whole person, completely replacing body parts and lost physiological functions rather than merely substituting for impaired structure and function. If a life-sustaining intervention can be considered a "replacement"-a part of the patient as a unified whole person-then it seems that deactivation is better classified as a case of killing rather than a case of forgoing a life-sustaining treatment. ICDs are not a "replacement" therapy in this sense. The deactivation of an ICD is best classified, under the proper conditions, as the forgoing of an extraordinary means of care. As technology becomes more sophisticated, however, and new interventions come to be best classified as "replacements" (a heart transplant would be a good example), "discontinuing" these interventions should be much more morally troubling for those clinicians who oppose euthanasia and assisted suicide.

KEY WORDS: implantable cardioverter defibrillators; ethics; withholding treatment.

J Gen Intern Med 23(Suppl 1):69-72

DOI: $10.1007 / \mathrm{s} 11606-007-0326-x$

(c) Society of General Internal Medicine 2007

I mplantable Cardioverter Defibrillators (ICDs) have proven 1 very effective in preventing sudden cardiac death. Medicare's decision to pay for these devices all but assures that their use will soon become widespread. ${ }^{1}$

Yet, like any technological innovation, these devices are not an unalloyed good. Under certain circumstances it seems best to discontinue treatment. For example, some patients receive shocks so frequently that it becomes extremely burdensome. If a Do Not Resuscitate order is written, whether on the basis of the patient's underlying cardiac disease or some comorbid condition, it may be senseless to continue ICD use.

Several previously published ethical analyses of the discontinuation of ICD treatment have noted that some persons believe that discontinuing an ICD is ethically different from discontinuing other treatments. These analyses, by and large, have declared such misgivings to be misguided and have proceeded to analyze the discontinuation of ICD treatment using standard bioethical categories such as patients' rights, refusal of unwanted therapy, autonomy, futility, and nonmaleficence. ${ }^{2-6}$ Nonetheless, it seems that cardiologists and patients have not read the bioethics literature. Cardiologists and their patients view the deactivation of an ICD as something special, and they discuss the possibility very infrequently.

The 2 articles by Goldstein et al. ${ }^{7,8}$ in this issue of JGIM are an important step toward understanding this discrepancy between ethical analysis and clinical reality. Broadly speaking, these articles demonstrate the significant contribution that qualitative studies can make to empirical research about ethics, delving more deeply into the meanings behind answers to survey questions. Specifically, these articles raise 2 important points for ethics. First, they show that issues long thought settled intellectually by ethicists, such as the difference between withholding and withdrawing life-sustaining treatments, still present lingering doubts for patients and practitioners. Patients and cardiologists alike seem to view implanting an ICD as a "bridge" that one crosses with no possibility of return. Second, these rich qualitative data suggest that there may be more going on here from a moral point of view than the ethics of the 1970s can handle. As technology progresses, ethics must keep pace.

What may be different about an ICD? Paola and Walker ${ }^{9}$ have suggested that the new wrinkle may be that the technology has been internalized-that an ICD may come to be viewed as having "become a part of the patient." Using the philosophical term for the study of being, ontology, they suggest that an external defibrillator is easier to forgo than one that is internal to the person, because the latter may be considered part of his or her being. Borrowing from the concepts of property law, they coin the term "biofixture" to describe technologies that have become part of a person. They accept the morality of the discontinuation of an ICD because they view this device as only partly a biofixture. They argue that a heart transplant, however, would be a real biofixture, and no one who is opposed to euthanasia would think it permissible to "deactivate" a transplanted heart.

This interesting foray into ontology and ethics raises many more questions than it answers, however. As biotechnological progress marches forward, new interventions are challenging our notions about the difference between killing and allowing 
to die. If one is opposed to euthanasia yet accepts the moral permissibility of discontinuing life-sustaining treatments under certain conditions, do new technologies, such as ICDs, require that the line between killing and allowing to die be redrawn? What is the "something intrinsic to the nature and function of these devices" 8 that causes some to consider them differently than other treatments?

\section{WITHHOLDING VS. WITHDRAWING}

The data from the articles by Goldstein et al. suggest that both cardiologists and their patients tend to think that once a device such as an ICD is in place, one ought not stop it. As a general rule, philosophers have suggested that the conditions under which one could justify withholding a treatment are those under which one could justify withdrawing a treatment. ICDs raise no new moral issues with respect to this question. For example, if the patient were irreversibly and imminently dying of a painful cancer and had recurrent ventricular tachycardia, one would be perfectly justified in not placing an ICD. Rationally, if the patient had an ICD implanted 2 years ago and now develops a painful cancer and death is imminent, deactivating the ICD seems just as justifiable as withholding it. What the studies of Goldstein at al. tell us, however, is that what seems equivalent according to the logic of ethics continues to feel psychologically different to both patients and practitioners. Clinicians must be sensitive to these feelings in dealing with concrete clinical cases.

\section{CONTINUOUS VS. INTERMITTENT?}

Does the fact that the ICD is required intermittently rather than continuously mark a moral difference? Certainly it has not seemed so with respect to other life-sustaining treatments. The discontinuation of an intermittent treatment such as hemodialysis, should it become burdensome, has been judged morally acceptable by persons holding a wide variety of ethical viewpoints. In fact, as one of the cardiologists interviewed by Goldstein at al. reports, the very fact that it functions only intermittently might make it psychologically easier to deactivate an ICD than to deactivate the pacemaker of a patient with complete heart block. ICDs raise no new ethical issues in this regard.

\section{DURATION}

Some might consider the duration of therapy morally important. But is this true? If the ICD in the case of the patient with cancer that I discussed above had been in place for 20 years instead of 2 , with no changes except for batteries, would the duration alone make us think that it would be immoral to deactivate it if the patient were imminently dying, in great pain, and the device might only prolong that state? It seems to me this would not be the case. Consider a patient who has been ventilator-dependent for 30 years after contracting polio, is not depressed, and comes to the conclusion, "I've had enough." Might we not be more willing to accept his request to discontinue the ventilator as a well thought out and morally acceptable choice than if the same request were made by the patient after only 1 week of ventilator support? Duration of therapy does not seem to be the morally decisive factor.

\section{REGULATIVE VS. CONSTITUTIVE?}

All therapies are restorative in intent, ${ }^{10}$ but different therapies restore patients in 2 broadly different ways. Some therapies are "regulative." That is to say they coax the body back toward its own homeostatic equilibrium. Antidysrhythmic drugs and antipyretics are regulative therapies in this sense. Other therapies are "constitutive." They take over a function that the body can no longer provide for itself. Pacemakers and insulin are constitutive therapies in this sense. Might this distinction mark a moral difference between killing patients and allowing them to die?

If anything, it would seem that the discontinuation of constitutive therapies would raise more questions than the discontinuation of regulative therapies. Mimicking physiology, doing what the body no longer can do for itself, seems closer to being "a part of the patient" than does a therapy that nudges the body into healing itself.

Despite the psychological differences that this distinction might raise, however, we still regularly accept the morality of discontinuing constitutive therapies such as ventilatory support. The fact that a treatment is constitutive does not seem to mark a moral difference between killing and allowing to die. Further, the fact that ICDs are regulative, not constitutive, suggests that this distinction cannot explain any special moral worries about deactivating an ICD.

\section{INTERNAL VS. EXTERNAL}

Does the fact that a technology has become internal to the body mark the boundary between killing and allowing to die? This question seems to come closer to explaining the concerns raised by the patients and practitioners about deactivating ICDs, but does it stand up to ethical analysis? Does the fact that many new medical technologies are inside the body mean that they have thereby become part of the person so that deactivating an ICD or a pacemaker becomes morally equivalent to discontinuing the function of a natural heart by injecting $\mathrm{KCl}$ ?

Elsewhere, ${ }^{11}$ I have defined killing as an act in which an agent performs an action that creates a new, nontherapeutic, lethal pathophysiological state in a human being with the intention of thereby causing that human being's death. By contrast, I have defined allowing to die as an act in which an agent either performs an action to remove a treatment for a preexisting fatal disease or refrains from action that would treat a preexisting fatal disease, either intending that this person should die by way of that act or not so intending. The acts of allowing to die that have traditionally been thought morally permissible are those in which the physician's intentions are to aim not at making the patient dead, but simply at stopping the treatment.

These definitions make no reference to internal or external. By these definitions, deactivating an external pacemaker is morally equivalent to deactivating an internal pacemaker, and 
deactivating an external defibrillator is morally equivalent to discontinuing an ICD.

But is this correct? Does not having a device inside a patient make it a part of the patient, part of her physiology, so that stopping its function is killing? Certainly, this intuition seems correct if one is talking about a heart transplant. Stopping the function of a transplanted heart with an injection of $\mathrm{KCl}$ seems morally no different from stopping a native heart with an injection of $\mathrm{KCl}$. And is not a heart transplant every bit as much a technological intervention as is an ICD?

Upon further reflection, the fact that a treatment is "inside" the body does not, of itself, seem to do the moral work some might think it does. Consider and compare the following technological interventions: an LHRH agonist implant for prostate cancer and a skin transplant after a severe burn. If one were to discontinue these therapies, however, the mere fact that one treatment is placed under the patient's skin, whereas the other is placed over the patient's skin does not constitute the difference between killing and allowing to die. If the patient with prostate cancer were experiencing hot flashes from the LHRH action of the drug and having pain at the site of the implant, his request to have it removed should be honored. But what if the burn patient were to ask that the skin transplant be removed, saying that she had grown tired of the need to take anti-rejection medication? Without an intact integument, the patient would experience sepsis and die. Most plastic surgeons would refuse to do this on the grounds that they would be mutilating, if not killing, the patient, even if she were otherwise dying from some other comorbid disease. The mere fact that a technological intervention has been placed under the skin does not seem to mark the moral difference between killing and allowing to die.

\section{REPLACEMENT VS. SUBSTITUTE THERAPY}

So, then, perhaps those who have approached the question as a standard application of the principles typically used in bioethics are right after all. Perhaps there is nothing special about devices such as ICDs.

Such a conclusion would be overly hasty. Jansen has argued that the questions raised by advances in medical technology such as ICDs are challenging our notions about the boundaries of the self. ${ }^{12}$ Particularly, as biotechnological treatments become more "bio" than "techno," we need to think clearly about the characteristics that render a treatment a part of the patient's self, so that its discontinuation is morally indistinguishable from killing. Jansen has offered 3 rules of thumb for helping to judge whether a particular technological intervention ought to be considered a part of the patient. She suggests that there are more serious grounds for considering the technological intervention a part of the patient if it is located inside the patient, has been in the patient for a long time, and plays something akin to what I have called a constitutive therapeutic role.

Certainly, none of these factors is, of itself, morally decisive. Jansen would not seem to disagree. I have explained above, however, why I believe none of these 3 factors can bear the weight of the distinction between killing and allowing to die.
And, whereas Jansen's insight is correct-that some treatments must truly be considered within the ontological boundaries of the patient's "self"-I do not think the criteria she has suggested fully capture the distinction. And while I further agree with her that these will necessarily be rules of thumb and that a "bright line" will be hard to draw, I think we can press for greater clarity.

I would like to suggest 2 alternative guiding principles that, while provisional hypotheses, might advance the discussion.

First, interventions that are regulative are never "self." These interventions are distinct from the organism and extrinsic to its function, whether administered inside or outside the body. They function by attempting to regulate bodily functions, coaxing them back toward homeostasis. An ICD is regulative. It does not supply the heart rhythm. Rather, it shocks an abnormal cardiac rhythm back into normal sinus rhythm. Although it is internal to the body, it is still not "self." One of the patients quoted by Goldstein et al. illustrates the psychology of distinguishing between ICD and self nicely when she describes how she talks to her ICD, treating it as something other than her "self" although it is located within her. ${ }^{7}$ Regulatory therapies, no matter how sophisticated, and whether located inside the body or not, can be thought about just as one would think about withholding or withdrawing more standard forms of therapy at the end of life. They may be forgone if they are futile or if the burdens of treatment become disproportionate to the benefits.

Second, some, but not all, constitutive therapies are distinct from the self. I am not certain how best to characterize this distinction, but my preliminary hypothesis is that we can distinguish between constitutive therapeutic interventions that have replaced the pathologically disordered function and those that are substitutes for the pathologically disordered function. The distinction between these 2 kinds of constitutive therapies will not always be clear, but I hope to make it clear enough that it can be clinically and morally useful. In my view, in the proper circumstances, it is morally permissible to withhold or withdraw substitutive therapies, but the more an intervention can be understood as a replacement therapy, the less it seems morally appropriate to withdraw it.

\section{HOW MIGHT REPLACEMENT AND SUBSTITUTIVE THERAPIES BE DISTINGUISHED?}

What I mean by a replacement therapy is a technological intervention that participates in the organic unity of the patient as an organism. This is what it really means to say that a technological intervention has become "a part of the patient." A replacement therapy is one that has become part of the patient's restored physiology. The most important feature of a replacement therapy is that it provides the function that has been pathologically lost, more or less in the same manner in which the patient was once able to provide this function when healthy. Thus, for instance, a renal transplant is a replacement therapy, whereas peritoneal dialysis (although it also takes place inside the body) is a substitutive therapy.

Additional signs suggestive of an intervention being a replacement therapy might include: (1) its responsiveness to 
changes in the organism or its environment, (2) properties such as growth and self-repair, (3) independence from external energy sources or supplies, (4) independence from external control by an expert, (5) immunologic compatibility, (6) physical integration into the patient's body. The paradigmatic replacement therapy is thus a well-functioning organ transplant from an identical twin. The more a technological intervention meets the conditions for being a replacement therapy, the harder it is to contend that it is extrinsic to the patient's identity.

This distinction between replacement and substitutive types of constitutive therapies might help us to say how far someone who is opposed to euthanasia could reasonably go in classifying certain cases as morally appropriate withdrawal of lifesustaining treatment rather than morally unacceptable cases of killing. Whereas there is no absolute standard for judging whether something is a replacement or a substitute, the more clearly a technology can be classified as a replacement therapy, the greater the case for judging that its discontinuation would constitute an immoral act of killing. Replacement therapies become part of the restored physiology of the patient, part of the integrated unity of the patient as an intact individual organism. To discontinue such therapies is better understood as introducing a new lethal pathophysiological state rather than discontinuing a treatment that is merely substituting for a preexisting lethal pathophysiological lack of that function. The discontinuation of a replacement therapy thus becomes an act of killing.

To illustrate, consider the fact that there are cases in which discontinuing insulin injections in a diabetic patient would be morally appropriate. If the patient were imminently dying of a malignancy, even the burdens of being injected with insulin or having finger-stick checks for blood glucose might be considered disproportionate to the benefits. We ought therefore to permit the discontinuation of insulin in some such cases. Insulin injections clearly represent a case of a constitutive rather than regulative therapy. The injections mimic the normal physiology. However, giving insulin by injection is a substitutive rather than replacement therapy. It provides the normal physiological function in an abnormal way and the treatment is not part of some new organic unity that has altered the identity of the patient.

Consider a slightly different case, however. If the same patient had previously undergone an islet cell transplant as a treatment for his diabetes, it would seem highly morally problematic to "discontinue" insulin therapy under these same end-of-life conditions by injecting streptozosin to kill the transplanted islet cells. Islet cell therapy is a replacement therapy and, as such, the injection of streptozocin would be mischaracterized if one were to describe it as the mere discontinuation of a life-sustaining therapy. The cells would have become part of the patient-an integrated aspect of her restored physiology-and to destroy these cells would be ethically indistinguishable from destroying the native islet cells of a healthy person.

\section{CONCLUSIONS}

Those who are opposed to euthanasia but supportive of the withholding and withdrawing disproportionately burdensome life-sustaining treatments need not demur at the idea of deactivating ICDs. The analysis I have presented shows that deactivating an ICD can be ethically distinguished from killing and considered a part of good palliative care. However, the argument necessary to reach this conclusion will doubtless prove challenging for persons unaccustomed to philosophical thinking. The data from Goldstein et al. describe in detail how much effort will be needed in making this argument clear for cardiologists and patients. It is critically important, however, that we begin thinking seriously and carefully about what makes an intervention a part of the patient, rather than a treatment that is extrinsic to the patient's self, even if it is located inside the patient's body. The rapid pace of technological progress assures us that these sorts of questions will continue to surface in clinical practice. Ethics, as the most practical branch of philosophy, must be prepared to keep pace with these challenges.

Potential Financial Conflict of Interest: None disclosed.

Corresponding Author: Daniel P. Sulmasy, OFM, MD, PHD; John J. Conley Department of Ethics, St. Vincent's Hospital-Manhattan, 153 W. 11th St., New York, NY 10011, USA (e-mail: daniel_sulmasy@nymc. edu).

\section{REFERENCES}

1. McClellan MB, Tunis SR. Medicare coverage of ICDs. N Engl J Med. 2005;352:222-4.

2. Lewis WR, Luebke DL, Johnson NJ, Harrington MD, Costantini O, Aulisio MP. Withdrawing implantable defibrillator shock therapy in terminally ill patients. Am J Med. 2006;119:892-6.

3. Braun TC, Hagen NA, Hatfield RE, Wyse DG. Cardiac pacemakers and implantable defibrillators in terminal care. J Pain Symptom Manage. 1999;18:126-31.

4. Berger JT. The ethics of deactivating implanted cardioverter defibrillators. Ann Intern Med. 2005; 142:631-4.

5. Mueller PS, Hook CC, Hayes DL. Ethical analysis of withdrawal of pacemaker or implantable cardioverter-defibrillator support at the end of life. Mayo Clin Proc. 2003;78:959-63.

6. Pellegrino ED. Decisions to withdraw life-sustaining treatment: a moral algorithm. JAMA. 2000;283:1065-7.

7. Goldstein NE, Mehta D, Siddiqui S, et al. Sean Morrison RS. That's like an act of suicide: patients' attitudes toward deactivation of implantable defibrillators. J Gen Intern Med DOI 10.1007/s11606-007-0239-8.

8. Goldstein NE, Mehta D, Teitelbaum E, Bradley EH, Sean Morrison RS. It's like crossing a bridge: complexities preventing physicians from discussing deactivation of implantable defibrillators at the end of life. J Gen Intern Med 2007 DOI 10.1007/s11606-007-0237-x.

9. Paola FA, Walker RM. Deactivating the implantable cardioverterdefibrillator: a biofixture analysis. South Med J. 2000;93:20-3.

10. Jansen LA, Sulmasy DP. Proportionality, terminal suffering and the restorative goals of medicine. Theor Med Bioethics. 2002;23:321-37.

11. Sulmasy DP. Killing and allowing to die: another look. J Law Med Ethics. 1998;26:55-64.

12. Jansen LA. Hastening death and the boundaries of the self. Bioethics. 2006;20:105-11. 